\title{
The feasibility analysis of omission of elective irradiation to level IB lymph nodes in low-risk nasopharyngeal carcinoma based on the 2013 updated consensus guideline for neck nodal levels
}

Xiaomin Ou ${ }^{1,2}$, Yibing Miao ${ }^{1,2}$, Xiaoshen Wang ${ }^{1,2}$, Jianhui Ding ${ }^{3}$, Xiayun $\mathrm{He}^{1,2}$ and Chaosu $\mathrm{Hu}^{1,2^{*}}$ (D)

\begin{abstract}
Background: Level IB metastasis is rare in nasopharyngeal carcinoma (NPC). The purpose of this study is to investigate the high-risk factors for level IB metastasis and evaluate the feasibility of omission of elective irradiation to level IB in the low-risk subgroups in NPC.

Methods: This retrospective study identified 532 patients with NPC treated by definitive radiation in our institution from 2009 to 2010. Level IB nodes were electively irradiated based on the physician's decision. Diagnostic head and neck MRIs were reviewed. The involvements of nodal levels were evaluated according to 2013 updated guidelines of RTOG. The correlations of level IB metastasis and other factors were studied using Chi-square test and logistic regression model. Log-rank tests were used to compare survival rates. Cox proportional-hazards models were used to evaluate the effect of various factors. Patient-reported xerostomia was recoded in every follow-up and the extents of delayed xerostomia at 1 year post-radiation were compared between those with/without elective level IB irradiation.
\end{abstract}

Results: N stage, bilateral nodal metastasis, level II involvement, level IIA involvement, level IIA with multiple levels involvement, maximal axial diameter (MAD) of level IIA nodes $>20 \mathrm{~mm}$, MAD of neck lymph nodes $>30 \mathrm{~mm}$, necrosis of level IIA nodes, extracapsular spread of level IIA correlated with level IB metastasis by univariate analysis. In multivariate analysis (MVA), bilateral nodal involvement, MAD of level IIA nodes $>20 \mathrm{~mm}$ or extracapsular spread of level IIA nodes, were independent predictive factors for level IB metastasis. Patients without either these factors were denoted low-risk group and the rest high-risk group. Of the low-risk group, there was no significant difference of regional control and overall survival (OS) between those with or without elective irradiation. The percentage of level IB recurrence of those without elective irradiation was $0.46 \%$. Elective level IB irradiation was not significant upon MVA both for regional control and OS. Of the high-risk group, elective level IB irradiation was marginal significant for regional control, but not for OS upon MVA. No regional recurrence located at level IB. Overall, omission of elective irradiation to level IB reduced the mean doses of submandibular glands, but did not improve patient-reported xerostomia.

Conclusion: For patients without high-risk factors of level IB metastasis, omission of elective level IB irradiation did not impair regional control and OS in NPC.

Keywords: Nasopharyngeal carcinoma, Elective nodal irradiation, Submandibular gland, Xerostomia, Late toxicity

\footnotetext{
* Correspondence: hucsu62@yahoo.com; hucsu62@163.com

'Department of Radiation Oncology, Fudan University Shanghai Cancer Center, Shanghai, China

${ }^{2}$ Department of Oncology, Shanghai Medical College, Shanghai, China

Full list of author information is available at the end of the article
} 


\section{Introduction}

The 5-year overall survival of nasopharyngeal carcinoma (NPC) has achieved $84 \%$ of non-metastatic cases with the use of intensity-modulated radiation therapy (IMRT) and optimal chemotherapy [1-4]. Since the survival is prolonged, the late toxicities merit more attention. The most frequently observed late complication was xerostomia. 39.3-40.1\% [4-6] of patients reported xerostomia at more than 1 year post-radiation. Sparing of saliva glands is crucial to reduce xerostomia. IMRT is superior to conventional two-dimensional radiotherapy in preserving parotid function and results in less severe delayed xerostomia [6]. Parotid-sparing IMRT for NPC preserved half of parotid excretion compared with baseline [7]. However, sparing of parotid alone has increased salivary flow rate [8], but inconsistently translated to improvement of patient-reported xerostomia [6, 8-10].

Parotids produce most saliva under stimulation, while the submandibular glands (SMG) contribute to the majority of unstimulated saliva [11]. SMG, together with minor salivary glands in the oral cavity, produce mucins that maintain subjective perception of hydration [10]. It is reported that SMG salivary flow rate depends on mean dose and recovers over time up to a threshold of 39Gy [12]. Restriction of mean dose of SMG less than 39Gy helps to improve observer-reported and patientreported xerostomia [9, 12, 13].

However, the SMG is often included in the target volume of NPC when level IB receives radiation. According to the protocol of RTOG 0225, clinical target volume (CTV) encompassed unilateral level I to level V. The routine of Prince of Wales Hospital in Hong Kong is to electively irradiate level IB to V with a total dose of 54$60 \mathrm{~Gy}$ for node-negative cases [14]. However, a number of institutions in mainland of China $[4,11,15]$ do not recommend routinely including level IB in the irradiation volume. Currently, there is no consensus of criteria on elective irradiation of level IB. Zhang et al. [11] has recently demonstrated that level IB-sparing IMRT should be feasible for patients without high risk factors for level IB metastasis. However, in this study, only a few factors were included in the analysis, such as $\mathrm{T}, \mathrm{N}$, level IIA and retropharyngeal metastasis, et al. More clinical characteristics should be incorporated in to the analysis for level IB metastasis, especially the details of involvement of various neck nodal levels. Hence, we performed this retrospective study, based on the elaborate analysis using the new delineation guidelines of nodal level involvement of Radiation Oncology Therapy Group (RTOG) [16]. The aim of this study is to investigate the high-risk factors for metastasis of level IB and evaluate the feasibility of omission of elective irradiation to level IB in the subgroups without any of the high-risk factors. The objective of this study is to define the criteria of level IB- sparing radiation, in order to protect the function of submandibular glands and minimize late toxicities.

\section{Materials and methods}

Inclusion or exclusion criteria

Institutional Review Board approval was obtained for this retrospective review of patients. Consecutive patients with newly diagnosed, non-metastatic NPC, with complete imaging and treatment planning data, treated with definite IMRT in our center, were deemed eligible. Exclusion criteria were as follow: (1) surgery to primary tumor or cervical nodes prior to radiation (except for nasopharyngeal biopsy or fine needle aspiration of nodal disease); (2) who failed to complete the whole course of radiation; (3) who did not perform pretreatment MRI of head and neck in our center or the imaging data of pretreatment MRI were not traceable; (4) the treatment plan data were unavailable.

\section{Imaging analysis}

One experienced radiologist and one experienced radiation oncologist in head and neck cancer reviewed all the imaging data. Any disagreement was solved by discussion. Cervical lymph nodes were defined as metastatic if the shortest axial diameter of jugulodigastric lymph node was $\geq 11 \mathrm{~mm}$, or that of other lymph nodes was $\geq 10 \mathrm{~mm}$, or there was a group of three or more lymph nodes in critical size [17]. The lateral retropharyngeal nodes were considered metastatic with their shortest diameter $\geq 5 \mathrm{~mm}$. Any visible node in the median retropharyngeal group was considered malignant $[18,19]$. In addition, the presence of extracapsular spread or central necrosis was also a sign of metastasis $[17,18,20]$. The involvement of nodal levels was evaluated according to the 2013 updated guidelines of RTOG [16]. All patients were restaged based on the 2010 AJCC staging system.

\section{Radiotherapy techniques}

Patients were immobilized in the supine position with a thermoplastic mask. CT was performed after immobilization, obtaining 3-mm slices from the anterior clinoid process to the hyoid bone, and $5-\mathrm{mm}$ slices from the hyoid bone to $2 \mathrm{~cm}$ below the sternoclavicular joint. The target volumes were outlined on each layer of the CT images on an IMRT workstation. The gross tumor volume (GTV) included primary tumor and metastatic lymph nodes. The details of the delineation of high-risk clinical target volume of primary tumor were described in our previous publication [4]. The high-risk CTV of cervical nodes should include bilateral coverage of levels II, III, VA and retropharyngeal nodes in N0 patients. For patients with metastatic cervical nodes of the upper neck (above cricoid cartilage), the low risk CTV should cover 
level IV and VB. For individuals with metastatic cervical nodes involving the lower neck, all the neck levels from II to V were defined as high-risk CTV. Elective level IB irradiation was decided by the attending physician, mainly based on these factors: maximal diameter of level IIA, extracapsular spread of level IIA, necrosis of level IIA, N stage, multiple levels involvement, maximal diameter of cervical nodes, as well as extracapsular spread or necrosis of cervical nodes. These factors were reasonably selected. The first three factors indicated the extent of involvement of level IIA, while the remaining reflected the burden of nodal metastasis and the risk of nodal relapse.

A margin of $3-5 \mathrm{~mm}$ around GTV and CTV should be added to account for the patient motion and set-up error. A simultaneous integrated boost method was used. 66 to 70.4 Gy was prescribed to primary tumor in 30-32 fractions. 66 Gy was delivered to the metastatic nodes in 30-32 fractions. The high-risk and low-risk CTV received 60 Gy and $54 \mathrm{~Gy}$, respectively.

SMGs were contoured, but the SMG doses were not constrained during inverse treatment planning. Given the vicinity of CTV60, one half of SMG was within the isodose line 39Gy even if the ipsilateral level IB was not included in the CTV (Additional file 1: Figure S1). The mean doses of SMG were recorded retrospectively.

\section{Follow-up}

Patients were followed up after completion of radiotherapy every 3 months in the first 2 years, then every 6 months from year 2 through year 5, and then annually. MRI of head and neck was required every 3 to 6 months in the first 3 years. Chest CT and abdominal sonography were performed at least every year. Bone scan or PET/ CT was done when clinical indicated. Delayed xerostomia was evaluated in every follow-up, according to the patient's subjective feeling and scored based on Common Terminology Criteria for Adverse Events 3.0 version grading system (CTCAE 3.0). Given that radiationinduced xerostomia faded with time and significantly recovered at 1 year post-IMRT [6], we compared the delayed xerostomia at 1 year after radiotherapy between the two arms - electively irradiation to level IB or not.

\section{Statistical analysis}

Kaplan-Meier overall survival (OS), distant metastasisfree survival (DMFS) and local recurrence-free survival (LRFS) and regional recurrence-free survival (RRFS) were calculated. Event times for death/recurrence were calculated from the first day of radiation. Viable tumor cells with the same histological subtype at the time of salvage neck dissection or fine-needle aspiration were defined as regional failure. Baseline comparison was performed using independent $t$ test and Chi-square test (or Fisher's exact test, if indicated). The correlation of level IB metastasis and other factors were studied using Chi-square test (or Fisher's exact test, if indicated). Logistic regression model (backward conditional) was used to investigate the independent predictive factor for level IB metastasis. In order to clarify the goodness of fit of Logistic model, we performed Hosmer-Lemeshow test. The $p$ value of Hosmer-Lemeshow test $>0.05$ indicated good estimation of Logistic model. Actual survival rates were compared by Log-rank test. Cox proportionalhazards models (enter method) were used to assess the effect of age, sex, $\mathrm{T}$ stage, $\mathrm{N}$ stage, nodal necrosis, chemotherapy, elective level IB radiation and boost irradiation to lymph nodes on regional control and overall survival. Since nodal extracapsular spread had certain correlation with elective level IB radiation, nodal extracapsular spread was used as classified factor in Cox model. Statistical significance was defined as $p<0.05$ based on two-sided tests. All analyses were performed by SPSS software, version 22.0.

\section{Results}

From January 2009 through December 2010, 869 consecutive patients with newly diagnosed, non-metastatic, biopsy-proven NPC completed the whole course of IMRT in our center. Among them, 46 patients received nodal excision prior to radiation, 185 patients underwent pretreatment MRI of nasopharynx or neck in other hospital and 106 patients were excluded due to data loss of pretreatment MRI or treatment plan backups. Therefore, the final study cohort consisted of 532 patients. The clinical characteristics were shown in Table 1 . With a median follow-up of 51.5 (1.0-74.7) months, 47 patients experienced local recurrence, 22 had regional failures and 61 developed distant metastasis. At the time of analysis, 68 patients were dead. The 5 -year estimated LRFS, RRFS and DMFS were $88.4 \%, 94.4 \%$ and $86.9 \%$, respectively. The 5 -year OS was $84.8 \%$.

\section{Risk factors of level IB metastasis}

Of 532 patients, $13(2.4 \%)$ presented with level IB metastasis. The correlation of level IB metastasis and other factors were investigated by Chi-square test (or Fisher's exact test, if indicated). More advanced $\mathrm{N}$ stage, bilateral nodal metastasis, level II involvement, level IIA involvement, level IIA metastasis with multiple levels involvement, the maximal axial diameter (MAD) of level IIA nodes $>20 \mathrm{~mm}$, MAD of neck lymph nodes $>30 \mathrm{~mm}$, necrosis of level IIA nodes, extracapsular spread of level IIA correlated with level IB metastasis $(p<0.05$, Table 2$)$. There was no significant association between level IB metastasis and nasal or oropharyngeal involvement. Similarly, of nodal positive patients $(n=450)$, more advanced $\mathrm{N}$ stage, bilateral nodal metastasis, level IIA involvement, MAD of level IIA nodes $>20 \mathrm{~mm}$, MAD of neck lymph 
Table 1 Patient characteristics $(n=532)$

\begin{tabular}{|c|c|c|c|}
\hline & & Number & Percentage (\%) \\
\hline \multirow[t]{2}{*}{ Gender } & Male & 390 & 73 \\
\hline & Female & 142 & 27 \\
\hline \multirow[t]{2}{*}{ Age } & Median & 50 & \\
\hline & Range & $11-80$ & \\
\hline Pathology & WHO II-III & 532 & 100 \\
\hline \multirow[t]{5}{*}{ Stage } & । & 39 & 7 \\
\hline & $\|$ & 179 & 34 \\
\hline & III & 179 & 34 \\
\hline & IVA & 55 & 10 \\
\hline & IVB & 80 & 15 \\
\hline \multirow[t]{4}{*}{$\mathrm{T}$} & $\mathrm{T} 1$ & 176 & 33 \\
\hline & $\mathrm{T} 2$ & 173 & 33 \\
\hline & T3 & 124 & 23 \\
\hline & $\mathrm{T} 4$ & 59 & 11 \\
\hline \multirow[t]{4}{*}{ N } & No & 82 & 15 \\
\hline & $\mathrm{N} 1$ & 241 & 45 \\
\hline & N2 & 129 & 24 \\
\hline & N3 & 80 & 15 \\
\hline \multirow[t]{2}{*}{ Elective IB Irradiation } & Yes & 232 & 44 \\
\hline & No & 300 & 56 \\
\hline \multirow[t]{2}{*}{ Radiation Boost } & Primary tumor & 32 & 6 \\
\hline & Nodal disease & 43 & 8 \\
\hline \multirow[t]{2}{*}{ Chemotherapy } & Yes & 451 & 85 \\
\hline & No & 81 & 15 \\
\hline
\end{tabular}

nodes $>30 \mathrm{~mm}$ and extracapsular spread of level IIA were associated with level IB metastasis (Table 2).

In multivariate analysis, bilateral nodal involvement $(p=0.045, \mathrm{HR}=4.056,95 \% \mathrm{CI}: 1.030-15.977), \mathrm{MAD}$ of level IIA nodes $>20 \mathrm{~mm}$ or extracapsular spread of level IIA nodes $(p=0.024, \mathrm{HR}=4.828$, 95\% CI: 1.227 18.996), were proven to be independent predictive factors of level IB metastasis at diagnosis (Table 3). Of nodal positive patients, MAD of level IIA nodes $>20 \mathrm{~mm}$ or extracapsular spread of level IIA nodes was demonstrated to be independent predictor of level IB metastasis by multivariate analysis $(p=0.034, \mathrm{HR}=4.287,95 \% \mathrm{CI}$ : $1.118-16.442$, Table 3). In order to clarify the goodness of fit of logistic model, we performed Hosmer-Lemeshow test. For all patients, the Pearson Chi-squared value was 0.317 and the $p$-value was 0.854 , which indicated the logistic model gave reliable estimation of observed incidence of level IB metastasis. Of node-positive patients, the Pearson Chi-squared value was 0.106 and the $p$-value was 0.948 , which indicated excellent prediction of logistic model.

\section{Low-risk group}

Based on previous analysis, the whole cohort of patients was divided into two subgroups. Those fulfilled either of risk factors (bilateral nodal involvement; MAD of level IIA nodes $>20 \mathrm{~mm}$ or extracapsular spread of level IIA nodes) were denoted high-risk group, while the rest were denoted low-risk group. Additional file: Table S1 compared the baseline characteristics by low-risk and highrisk groups.

Of the low-risk group (283), 216 did not received elective irradiation of level IB. Of note, patients with elective irradiation had more advanced $\mathrm{N}$ stage, higher proportion of nodal necrosis and extracapsular spread, higher proportion of chemotherapy, compared with those without elective irradiation (Additional file 1: Table S1). During the follow-up, four patients had neck relapse. Three of them did not receive level IB irradiation and only one recurrence located at level IB. The distributions of regional recurrence were shown in Additional file 1: Table S2. The percentage of level IB recurrence of those without elective irradiation was only $0.46 \%$. There was no significant difference of regional control (98.4\% vs. 97.4\%, $p=0.921$, Fig 1a), local control (90.4\% vs. $90.4 \%, p=0.599)$, DMFS $(96.5 \%$ vs. $91.5 \%$, $p=0.527)$ and OS (89.1\% vs.90.5\%, $p=0.798$, Fig $1 \mathrm{~b})$ between those with or without elective irradiation of level IB. Elective level IB irradiation was not significant upon multivariate analysis both for regional control and overall survival, after adjusting the effect of age, gender, $\mathrm{T}$ stage, $\mathrm{N}$ stage, nodal necrosis, chemotherapy and boost irradiation to nodes (Table 4). $\mathrm{N}$ stage was an independent prognostic factor for regional control.

In the subgroup of node-positive patients, no significant difference of 5-year regional control $(98.3 \%$ vs. $96.0 \%, p=0.798)$, local control $(89.9 \%$ vs. 90.0\%, $p=0.636)$, DMFS (96.3\% vs. $92.8 \%, p=0.677)$ and OS ( $88.4 \%$ vs. $91.5 \%, p=0.547$ ) was observed between those with or without elective level IB irradiation. Elective level IB irradiation was not significant upon multivariate analysis both for regional control and overall survival (Table 5). $\mathrm{N}$ stage was an independent prognostic factor for regional control and $\mathrm{T}$ stage was the independent prognostic factor for overall survival.

\section{High-risk group}

Of the high-risk group (249), 84 did not received elective irradiation of level IB. It was noteworthy that patients with elective irradiation of level IB had a higher proportion of nodal necrosis and extracapsular spread. During the follow-up, there were 18 nodal recurrences. However, the majority $(17 / 18)$ of them belonged to those with elective irradiation and no relapse located at level IB (Additional file 1: Table S2). Those with elective irradiation of level IB tended to have a poorer regional 
Table 2 Univariate analysis of risk factors of level IB metastasis in whole cohort and node-positive patients

\begin{tabular}{|c|c|c|c|c|c|c|c|}
\hline & \multicolumn{7}{|c|}{ Level IB metastasis at diagnosis } \\
\hline & \multicolumn{4}{|c|}{ All patients } & \multicolumn{3}{|c|}{ Node + patients } \\
\hline & Variable & $(-), n=519$ & $(+), n=13$ & $p$ value & $(-), n=437$ & $(+), n=13$ & $p$ value \\
\hline \multirow[t]{4}{*}{ N stage } & No & 82 & 0 & $0.011^{*}$ & 0 & 0 & $0.029^{*}$ \\
\hline & N1 & 238 & 3 & & 238 & 3 & \\
\hline & N2 & 121 & 8 & & 121 & 8 & \\
\hline & N3 & 78 & 2 & & 78 & 2 & \\
\hline \multirow[t]{2}{*}{ Bilateral nodal metastasis } & No & 350 & 3 & $0.002^{*}$ & 268 & 3 & $0.005^{*}$ \\
\hline & Yes & 169 & 10 & & 169 & 10 & \\
\hline \multirow[t]{2}{*}{ Nasal cavity involvement } & No & 402 & 10 & 1.000 & 342 & 10 & 1.000 \\
\hline & Yes & 117 & 3 & & 95 & 3 & \\
\hline \multirow[t]{2}{*}{ Oropharyngeal involvement } & No & 491 & 13 & 1.000 & 409 & 13 & 1.000 \\
\hline & Yes & 28 & 0 & & 28 & 0 & \\
\hline \multirow[t]{2}{*}{ Level II involvement } & No & 129 & 0 & $0.045^{*}$ & 47 & 0 & 0.378 \\
\hline & Yes & 390 & 13 & & 390 & 13 & \\
\hline \multirow[t]{2}{*}{ Level IIA involvement } & No & 298 & 0 & $0.000^{*}$ & 216 & 0 & $0.000^{*}$ \\
\hline & Yes & 221 & 13 & & 221 & 13 & \\
\hline \multirow[t]{2}{*}{ Level IIB involvement } & No & 156 & 2 & 0.362 & 74 & 2 & 1.000 \\
\hline & Yes & 363 & 11 & & 363 & 11 & \\
\hline \multirow[t]{2}{*}{ Level III involvement } & No & 257 & 4 & 0.182 & 175 & 4 & 0.501 \\
\hline & Yes & 262 & 9 & & 262 & 9 & \\
\hline \multirow[t]{2}{*}{ Level VA involvement } & No & 432 & 12 & 0.705 & 350 & 12 & 0.478 \\
\hline & Yes & 87 & 1 & & 87 & 1 & \\
\hline \multirow[t]{2}{*}{ Level IVA involvement } & No & 461 & 11 & 0.649 & 379 & 11 & 0.687 \\
\hline & Yes & 58 & 2 & & 58 & 2 & \\
\hline \multirow[t]{2}{*}{ Level IVB involvement } & No & 508 & 13 & 1.000 & 426 & 13 & 1.000 \\
\hline & Yes & 11 & 0 & & 11 & 0 & \\
\hline \multirow[t]{2}{*}{ Level VB involvement } & No & 509 & 13 & 1.000 & 427 & 13 & 1.000 \\
\hline & Yes & 10 & 0 & & 10 & 0 & \\
\hline \multirow[t]{2}{*}{ Level VC involvement } & No & 514 & 13 & 1.000 & 432 & 13 & 1.000 \\
\hline & Yes & 5 & 0 & & 5 & 0 & \\
\hline \multirow[t]{2}{*}{ Retropharyngeal nodal (Level VIIA)metastasis } & No & 252 & 4 & 0.205 & 108 & 2 & 0.738 \\
\hline & Yes & 267 & 9 & & 326 & 10 & \\
\hline \multirow[t]{2}{*}{ Level VIIB involvement } & No & 518 & 13 & 1.000 & 436 & 13 & 1.000 \\
\hline & Yes & 1 & 0 & & 1 & 0 & \\
\hline \multirow[t]{2}{*}{ Level VIII involvement } & No & 507 & 13 & 1.000 & 425 & 13 & 1.000 \\
\hline & Yes & 12 & 0 & & 12 & 0 & \\
\hline \multirow[t]{2}{*}{ Multiple levels involvement (III III, VA, IVA, VIII) } & $<2$ & 251 & 4 & 0.210 & 169 & 4 & 0.774 \\
\hline & $\geq 2$ & 268 & 9 & & 268 & 9 & \\
\hline \multirow{2}{*}{$\begin{array}{l}\text { Level IIA metastasis with multiple levels } \\
\text { involvement (II, III, VA, IVA, VIII) }\end{array}$} & No & 355 & 4 & $0.007^{*}$ & 273 & 4 & $0.038^{*}$ \\
\hline & Yes & 164 & 9 & & 164 & 9 & \\
\hline \multirow[t]{2}{*}{ MAD of IIA (mm) } & $\leq 20$ & 388 & 4 & $0.001^{*}$ & 306 & 4 & $0.005^{*}$ \\
\hline & $>20$ & 131 & 9 & & 131 & 9 & \\
\hline
\end{tabular}


Table 2 Univariate analysis of risk factors of level IB metastasis in whole cohort and node-positive patients (Continued)

\begin{tabular}{|c|c|c|c|c|c|c|c|}
\hline \multirow[t]{2}{*}{ MAD of ॥A (mm) } & $\leq 30$ & 479 & 11 & 0.274 & 397 & 11 & 0.346 \\
\hline & $>30$ & 40 & 2 & & 40 & 2 & \\
\hline \multirow[t]{2}{*}{ MAD of neck nodes(mm) } & $\leq 30$ & 288 & 3 & $0.020^{*}$ & 206 & 3 & 0.086 \\
\hline & $>30$ & 231 & 10 & & 231 & 10 & \\
\hline \multirow[t]{2}{*}{ Necrosis of level IIA nodes } & No & 463 & 9 & $0.048^{*}$ & 381 & 9 & 0.081 \\
\hline & Yes & 56 & 4 & & 56 & 4 & \\
\hline \multirow[t]{2}{*}{ ES of level IIA nodes } & No & 398 & 4 & $0.001^{*}$ & 316 & 4 & $0.003^{*}$ \\
\hline & Yes & 121 & 9 & & 121 & 9 & \\
\hline \multirow[t]{2}{*}{ MAD of $\| \mathrm{A}>20 \mathrm{~mm}$ or $\| \mathrm{A}$ necrosis } & No & 373 & 4 & $0.003^{*}$ & 291 & 4 & $0.014^{*}$ \\
\hline & Yes & 146 & 9 & & 146 & 9 & \\
\hline \multirow[t]{2}{*}{ MAD of $\| \mathrm{A}>20 \mathrm{~mm}$ or $\| \mathrm{A} E S$} & No & 363 & 3 & $0.001^{*}$ & 281 & 3 & $0.006^{*}$ \\
\hline & Yes & 156 & 10 & & 156 & 10 & \\
\hline
\end{tabular}

Abbreviation: $M A D$ maximal axial diameter, ES extracapsular spread ${ }^{*} p<0.05$

control (87.3\% vs. $98.1 \%, p=0.018)$. There was no significant difference of local control $(86.0 \%$ vs. $84.5 \%$, $p=0.108)$, DMFS (81.8\% vs. $78.0 \%, p=0.465)$ and OS (81.2\% vs.72.4\%, $p=0.156)$ between those with or without elective irradiation of level IB. Elective level IB irradiation was marginal significant for regional control, but not for overall survival, upon multivariate analysis incorporating age, gender, $\mathrm{T}$ stage, $\mathrm{N}$ stage, nodal necrosis, chemotherapy and boost irradiation to nodes. $\mathrm{N}$ stage and chemotherapy were demonstrated to be independent prognostic factor for overall survival (Table 4).

\section{Dose of SMG and delayed xerostomia}

Elective irradiation of level IB increased the mean dose of SMG, regardless of left $(6317+/-526$ vs. $4576+/-$ 2232 cGy, $p=0.000)$ or right side $(6368+/-210$ vs. $4610+/-2327$ cGy, $p=0.000)$. The mean dose of SMG exceeded 50Gy in above $75 \%$ (78.8\%, left SMG; $79.1 \%$, right SMG) of cases without ipsilateral level IB irradiation. In terms of delayed xerostomia, there was

Table 3 Multivariate analysis of risk factors of level IB metastasis by Logistic regression model

\begin{tabular}{llll}
\hline Variable & Significance & HR & $95 \% \mathrm{Cl}$ \\
\hline All patients $(n=532)$ & & & \\
Bilateral nodal involvement & $0.045^{*}$ & 4.056 & $1.030-15.977$ \\
MAD of IIA >20 mm or IIA ES & $0.024^{*}$ & 4.828 & $1.227-18.996$ \\
Nodal positive patients $(n=450)$ & & & \\
$\quad$ Bilateral nodal involvement & 0.062 & 3.601 & $0.938-13.826$ \\
MAD of IIA $>20$ mm or IIA ES & $0.034^{*}$ & 4.287 & $1.118-16.442$
\end{tabular}

Five variables with a $p$-value $<0.05$ in the univariate analysis of the whole cohort and node-positive cohort were included into Logistic regression model. The five variables are $\mathrm{N}$ stage, bilateral nodal involvement, MAD of IIA $>20 \mathrm{~mm}$ or IIA ES, MAD of IIA $>20 \mathrm{~mm}$ or necrosis of IIA, Level IIA metastasis with multiple levels involvement

${ }^{*} p<0.05$. Abbreviation: MAD maximal axial diameter; ES extracapsular spread no significant correlation between elective level IB irradiation and the grade of patient-reported xerostomia at 1 year post-IMRT ( $p=0.296$, Additional file 1: Table S3).

\section{Discussion}

Lymph node metastasis in NPC follows an orderly pattern along deep cervical lymph nodes. The most commonly involved regions include level II (70-87.4\%) and retropharyngeal lymph nodes (69-75.1\%), followed by level III, level IV and level V [21, 22]. The probability of "skip" metastasis is quite low (varies between 0.5-7.9\%) [21]. The incidence of level IB metastasis in NPC is very low, varies from $2.2 \%$ to $4.3 \%[18,20-22]$ (the rate of our study was $2.4 \%$ ). Level IB nodes receive efferent lymphatic from the level IA, the lower nasal cavity, the hard and soft palate, the maxillary and mandibular alveolar ridges, the cheek, the upper and lower lips, and most of the anterior tongue [16]. Level IB nodes do not receive direct drainage from nasopharynx. Hence, it is reasonable not to include level IB in the CTV of all patients with NPC.

In recent study including 3100 cases of NPC [22], all patients with level IB node involvement were simultaneously accompanied with level II lymphadenopathy. It is reasonable to assume that the enlargement of level II nodes blocks the routine pathway and causes reflux to level IB. Several studies [11, 23] further proved this hypothesis. Yuan et al. [23] reported that the diameter of level II was the only significant factor associated with level IB lymphadenopathy in multivariate analysis in NPC. Zhang et al. [11] demonstrated that the diameter of level IIA $>20 \mathrm{~mm}$ or extracapsular spread of level IIA was an independent predictive factor for level IB metastasis. In consistence with previous study, our research indicated that the diameter of level IIA $>20 \mathrm{~mm}$ or extracapsular spread of level IIA was significantly 


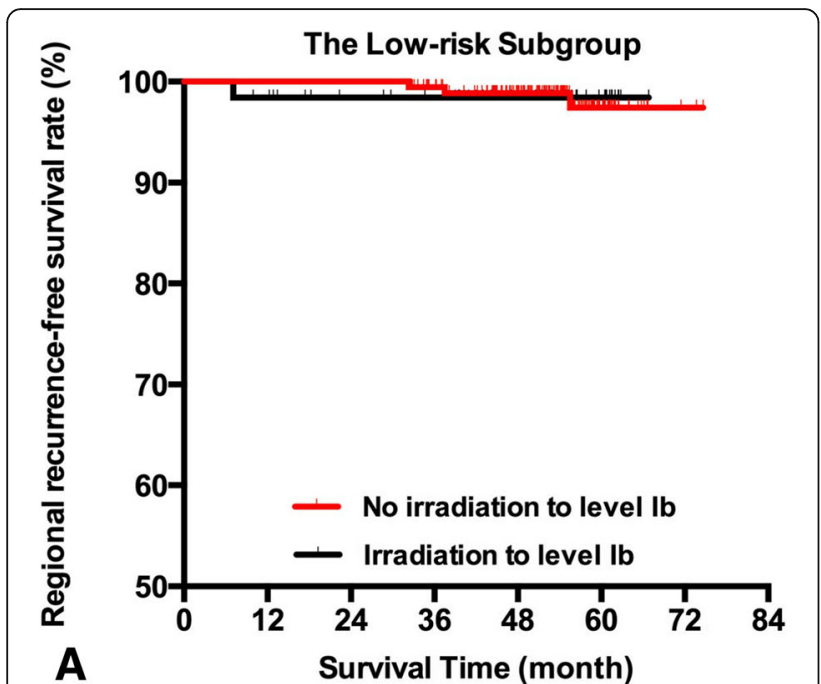

No. at risk

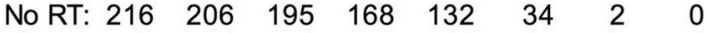

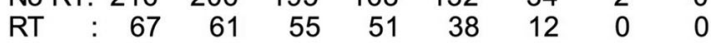

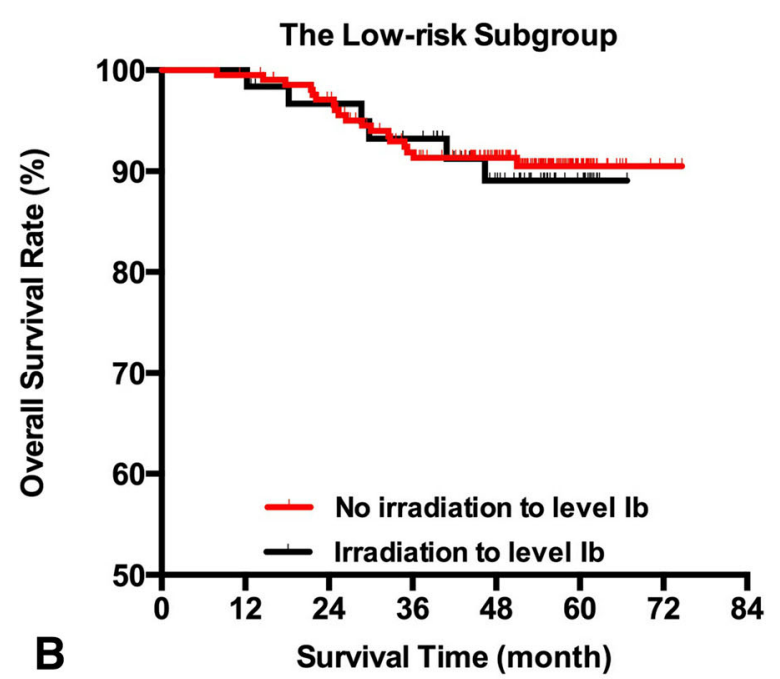

No. at risk

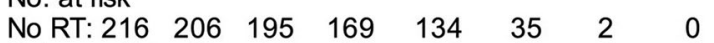

RT : $\begin{array}{llllllll}67 & 62 & 56 & 52 & 39 & 13 & 0 & 0\end{array}$

Fig. 1 Regional recurrence-free survival (a) and overall survival (b) compared by elective irradiation to level IB in low-risk patients with nasopharyngeal carcinoma. There wasn't significant difference of regional control $(98.4 \%$ vs. $97.4 \%, p=0.921)$ or overall survival (89.1\% vs.90.5\%, $p=0.798$ ) between those with or without elective irradiation to level IB. The estimated 5-year RRFS for those with/without elective irradiation was 65.9 months (95\% Cl: 64.0-67.7 months), 73.9 months (95\% Cl: $73.1-74.6$ months), respectively. The estimated 5 -year OS for those with/without elective irradiation was 62.8 months (95\% Cl: 59.7-65.9 months), 70.3 months (95\% Cl: 68.3-72.2 months), respectively

associated with level IB lymphadenopathy. The extracapsular spread causes involvement of carotid sheath, which was proven to correlate with level IB metastasis in another study by Yi et al. [24]. Taken together, the remarkable enlargement and spread of level IIA may result in blockage of routine drainage pathway, which causes reflux to its afferent lymph node-level IB. Bilateral involvement of cervical nodes were proven to be independent factor both in the present study and the report by Zhang et al. [11] as well. Given the possibilities of bilateral lymphatic drainage when nasopharynx tumor crossed the midline, bilaterally lymphadenopathy further added to burden of level II, therefore, increased the possibility of reflux to level IB.

The correlation between oropharyngeal involvement and level IB lymphadenopathy was inconsistently reported. Oropharyngeal involvement was not an independent predictive factor in the study of $\mathrm{Yi}$ [24] and Yuan [24] et al., but was significant in the report by Zhang [11] et al. Wang [22] et al. reported that about one third of cases with level IB involvement accompanied with oropharyngeal infiltration. In the present study, oropharynx invasion was not significantly associated with level IB metastasis. It is probably due to the limited number of cases with oropharyngeal involvement and the underpowered test. The correlation between oropharyngeal involvement and level IB lymphadenopathy warrants further investigation. In addition, the nasal cavity involvement was not an independent predictive factor for level IB metastasis in the present study, which was similarly observed in previous studies $[11,23,24]$. It is generally accepted that level IB receives lymphatic drainage of the anterior one third of nasal cavity $[24,25]$. However, tumor generally involves posterior one third of nasal cavity in NPC.

Furthermore, our study incorporated elaborate analysis of various neck nodal involvements into the risk-analysis of level IB metastasis, using the updated delineation guidelines of neck nodal levels [16], which was not reported in previous analyses $[11,23]$. Our study revealed that level IIA accompanying with multiple adjacent levels involvement was associated with level IB metastasis in univariate analysis, but did not reach statistically significant level in multivariate analysis. Similarly, in another investigation [23], four or more lymphatic drainage regions involved reached a marginal level $(p=0.05)$ in univariate analysis, but was not at statistically significant level in multivariate analysis. In addition, Yi et al. [24] demonstrated that level II/III/IV involvement was independent risk factor of level IIA metastasis in a cohort of patients treated with three-dimensional conformal radiotherapy. Given that multiple adjacent nodal levels involvement may block the level IIA efferent lymphatic drainage, it is reasonable to postulate that this may cause increased risk of reflux to level IB. Further investigation with a larger sample size is requested to confirm this postulation. Hence, we recommend taking this factor into consideration when evaluating the metastatic risk of level IB. 
Table 4 Multivariate analyses of factors on regional recurrence-free survival and overall survival (the whole cohort of patients)

\begin{tabular}{|c|c|c|c|c|c|c|c|c|}
\hline \multirow[t]{2}{*}{ Factor } & \multicolumn{4}{|c|}{ Regional control } & \multicolumn{4}{|c|}{ Overall survival } \\
\hline & $P$ value & $\mathrm{HR}$ & \multicolumn{2}{|l|}{$95 \% \mathrm{Cl}$} & $P$ value & $\mathrm{HR}$ & \multicolumn{2}{|l|}{$95 \% \mathrm{Cl}$} \\
\hline \multicolumn{9}{|l|}{ Low-risk group } \\
\hline Age $\left(\leq 50 y_{i}>50 y\right)$ & 0.500 & 0.471 & 0.053 & 4.208 & 0.068 & 2.394 & 0.938 & 6.109 \\
\hline Gender (M/F) & 0.760 & 0.657 & 0.045 & 9.654 & 0.920 & 0.956 & 0.392 & 2.331 \\
\hline $\mathrm{T}(\mathrm{T} 1 / \mathrm{T} 2 / \mathrm{T} 3 / \mathrm{T} 4)$ & 0.232 & 2.262 & 0.593 & 8.624 & 0.131 & 1.396 & 0.905 & 2.153 \\
\hline N (NO/N1/N2/N3) & $0.008^{*}$ & 12.076 & 1.927 & 75.675 & 0.925 & 0.966 & 0.472 & 1.980 \\
\hline Nodal necrosis (no/yes) & 0.722 & 0.530 & 0.016 & 17.542 & 0.147 & 2.155 & 0.763 & 6.088 \\
\hline Chemotherapy (no/yes) & 0.182 & 0.101 & 0.003 & 2.924 & 0.528 & 0.699 & 0.229 & 2.128 \\
\hline Elective IB irradiation (no/yes) & 0.941 & 1.122 & 0.054 & 23.274 & 0.799 & 1.143 & 0.408 & 3.206 \\
\hline Boost irradiation of lymph nodes (no/yes) & 0.993 & 0.000 & 0.000 & not reach & 0.888 & 1.162 & 0.145 & 9.340 \\
\hline \multicolumn{9}{|l|}{ High-risk group } \\
\hline Age $\left(\leq 50 y_{;}>50 y\right)$ & 0.198 & 1.909 & 0.714 & 5.104 & 0.058 & 1.798 & 0.980 & 3.299 \\
\hline Gender (M/F) & 0.682 & 0.768 & 0.217 & 2.715 & 0.506 & 0.785 & 0.385 & 1.602 \\
\hline $\mathrm{T}(\mathrm{T} 1 / \mathrm{T} 2 / \mathrm{T} 3 / \mathrm{T} 4)$ & 0.648 & 0.879 & 0.504 & 1.531 & 0.285 & 1.190 & 0.865 & 1.637 \\
\hline N (NO/N1/N2/N3) & 0.558 & 1.229 & 0.617 & 2.449 & $0.004^{*}$ & 2.080 & 1.269 & 3.411 \\
\hline Nodal necrosis (no/yes) & 0.971 & 1.019 & 0.365 & 2.843 & 0.725 & 1.130 & 0.573 & 2.226 \\
\hline Chemotherapy (no/yes) & $0.003^{*}$ & 0.029 & 0.003 & 0.299 & $0.000^{*}$ & 0.034 & 0.009 & 0.132 \\
\hline Elective IB irradiation (no/yes) & $0.045^{*}$ & 8.540 & 1.050 & 69.473 & 0.243 & 0.690 & 0.371 & 1.285 \\
\hline Boost irradiation of lymph nodes (no/yes) & $0.011^{*}$ & 3.793 & 1.351 & 10.645 & 0.906 & 1.058 & 0.413 & 2.710 \\
\hline
\end{tabular}

*Indicated $p<0.05$

Given the low incidence of metastasis of level IB in $\mathrm{NPC}$, it is reasonable not to include level IB in the CTV in the low-risk subgroup of level IB metastasis. A few studies have evaluated the feasibility of omitting irradiation to level IB in NPC. Chen et al. [26] retrospectively investigated a cohort of 120 patients treated with level IB-spared IMRT. During 54 months of follow-up, four patients had regional recurrence and none located in level IB. However, the details of cervical nodal involvement were not mentioned in this study. Zhang et al. [11] investigated a cohort of 1438 patients treated with IMRT and demonstrated the high-risk factors for level IB lymphadenopathy included the diameter of level IIA nodes $\geq 20 \mathrm{~mm}$ and/or level IIA nodes with extracapsular spread, positive bilateral nodes or oropharynx involvement at diagnosis. Patients without these risk factors were defined low-risk and none of them (0/904) experienced regional recurrence at level IB. Level IB irradiation was not an independent prognostic factor for locoregional control, distant metastasis and overall survival in low-risk subgroup. Similarly, Yi et al. [24] developed a risk scoring models to predict level IB metastasis and divided patients into low-risk and high-risk subgroups. The model included factors such as the carotid

Table 5 Multivariate analyses of factors on regional recurrence-free survival and overall survival (node-positive patients of low-risk group)

\begin{tabular}{|c|c|c|c|c|c|c|c|c|}
\hline \multirow[t]{2}{*}{ Factor } & \multicolumn{4}{|c|}{ Regional control } & \multicolumn{4}{|c|}{ Overall survival } \\
\hline & $P$ value & $\mathrm{HR}$ & $95 \% \mathrm{Cl}$ & & $P$ value & $\mathrm{HR}$ & $95 \% \mathrm{Cl}$ & \\
\hline \multicolumn{9}{|l|}{ Low-risk group } \\
\hline Age $\left(\leq 50 y_{i}>50 y\right)$ & 0.486 & 0.461 & 0.052 & 4.077 & 0.707 & 1.225 & 0.426 & 3.521 \\
\hline Gender (M/F) & 0.735 & 0.630 & 0.044 & 9.121 & 0.920 & 1.057 & 0.361 & 3.092 \\
\hline $\mathrm{T}(\mathrm{T} 1 / \mathrm{T} 2 / \mathrm{T} 3 / \mathrm{T} 4)$ & 0.298 & 2.064 & 0.527 & 8.089 & $0.046^{*}$ & 1.776 & 1.010 & 3.125 \\
\hline$N(\mathrm{~N} 1 / \mathrm{N} 2 / \mathrm{N} 3)$ & $0.015^{*}$ & 10.370 & 1.577 & 68.215 & 0.302 & 1.578 & 0.663 & 3.755 \\
\hline Nodal necrosis (no/yes) & 0.718 & 0.535 & 0.018 & 15.953 & 0.143 & 2.267 & 0.758 & 6.782 \\
\hline Chemotherapy (no/yes) & 0.177 & 0.105 & 0.004 & 2.760 & 0.295 & 0.449 & 0.100 & 2.009 \\
\hline Elective IB irradiation (no/yes) & 0.971 & 1.056 & 0.055 & 20.353 & 0.452 & 1.547 & 0.496 & 4.832 \\
\hline Boost irradiation of Lymph nodes (no/yes) & 0.993 & 0.000 & 0.000 & not reach & 0.773 & 1.374 & 0.158 & 11.972 \\
\hline
\end{tabular}


sheath involvement, maximal diameter of the neck lymph node $(\geq 20 \mathrm{~mm})$ and involvement of level II/III/ IV lymph nodes. In the low-risk group, level IB irradiation had no significant influence on overall survival, locoregional control and distant metastasis. Of note, patients in the prognostic study received threedimensional conformal radiotherapy [24].

In consistence with previous studies, our research defined the low-risk group as those without any of the following criteria: the diameter of level IIA nodes $>20 \mathrm{~mm}$ and/or level IIA nodes with extracapsular spread, positive bilateral cervical lymph nodes. In the low-risk subgroup without level IB irradiation, only one patient experienced regional recurrence at level IB. The percentage of level IB recurrence of those treated with level IB-sparing IMRT was only $0.46 \%(1 / 216)$. Level IB irradiation has no significant impact on regional control, distant metastasis and overall survival in the low-risk patients.

Interestingly, of the high-risk subgroup, those with elective irradiation of level IB tended to have a poorer regional control. Further analysis revealed that those with elective irradiation of level IB had a higher percentage of nodal necrosis and extracapsular spread, as well as greater maximal diameter of cervical nodes (data not shown, $43.06+/-16.76$ vs. $38.58+/-17.07 \mathrm{~mm}$, $p=0.049$ ). Nodal necrosis [27] and maximal diameter $[28,29]$ were proven to be prognostic factor for regional relapse, thus partially explaining the intriguing results. In addition, level IB was electively included in the CTV by the attending physician's decision, based on clinical and imaging characteristics. Therefore, patients with more adverse prognostic factors, tended to be treated with level IB irradiation.

In the present study, elective omission of level IB irradiation significantly reduced the mean dose of ipsilateral SMG, however, did not transfer to improvement of patient-reported xerostomia. One explanation is that there was no mandatory restriction of SMG dose during inverse planning in our institution at that time. According to a dose-effect study, the incidence of grade IV xerostomia dramatically increases once the mean dose of SMG exceeds a threshold of 39Gy. The saliva secretion of SMG could recover over time if the mean dose of SMG is below 39Gy [12]. Once the mean dose of SMG exceeds $50 \mathrm{~Gy}$, the probability of grade IV toxicity is above $80 \%$ [12]. In our study, more than $78 \%$ of patients received more than $50 \mathrm{~Gy}$ to SMG even if the ipsilateral level IB was not included in the radiation volume. Thus, despite elective omission radiation of level IB, the improvement of patient-reported xerostomia was mild. Another reason is that several factors have influence on xerostomia, such as bilateral involvement of level II, oropharyngeal infiltration of bulky tumor, mean dose of parotid, mean dose of oral cavity where minor salivary glands disperse. Gensheimer et al. [9] has shown that with careful dose constraint of parotid (mean dose $<24$ Gy) and contralateral SMG (mean dose <39 Gy), the contralateral SMG-sparing technique caused a notable and durable improvement of xerostomia. A future validation study that restrains the dose of parotid and spares SMG is warranted to confirm the benefit of elective omission of level IB in NPC.

A limitation of our study was the retrospective design. The incidence of level IB metastasis at diagnosis was relatively low. However, multivariate analysis indicated the significant risk factors for level IB involvement, which were well consistent with previous studies. Another limitation was no routine dose constraint for spared SMG in our institution at that time, which hampered the translation into the improvement of patientsubjective xerostomia.

\section{Conclusion}

In conclusion, our study demonstrated the risk factors for level IB metastasis were the diameter of level IIA nodes $>20 \mathrm{~mm}$ and/or level IIA nodes with extracapsular spread, positive bilateral cervical lymph nodes. For patients without either of the risk factors, omission of elective irradiation to level IB did not result into significant difference of regional control, DMFS and OS, but notably reduced the mean dose of ipsilateral SMG. It may be feasible to omit the radiation of level IB in the patients with low risk of level IB metastasis. A prospective study with careful dose constraint of SMGs and parotid is warranted to confirm the benefit.

\section{Additional file}

Additional file 1: Table S1. Comparison of clinical factors between highrisk and low-risk subgroups. Table S2. Distribution of regional recurrence by risk-stratified subgroups. Table S3. The grades of xerostomia by elective irradiation to level IB. Figure S1. Isodose distribution of submandibular glands. (PDF $237 \mathrm{~kb}$ )

\begin{abstract}
Abbreviations
CTV: Clinical tumor volume; DMFS: Distant metastasis-free survival; GTV: Gross tumor volume; IMRT: Intensity-modulated radiation therapy; LRFS: Local recurrence-free survival; MAD: Maximal axial diameter; NPC: Nasopharyngeal carcinoma; OS: Overall survival; RRFS: Regional recurrence-free survival; SMG: Submandibular gland
\end{abstract}

\section{Acknowledgements}

We thank our colleagues Dr. Zhou Xin, Dr. Shi Qi, Dr. Xing Xing, and Dr. Yang Youqi for their help of collecting clinical data.

\section{Funding}

This project was funded by the National Natural Science Foundation of China (Grant No. 81602372).

Availability of data and materials

The datasets used and/or analyzed during the current study are available from the corresponding author on reasonable request. 


\section{Authors' contributions}

Ou XM participated in the design of the study, performed data collection and analysis, and drafted the manuscript along with Hu CS. Miao YB collected the physics data of treatment planning. Wang XS, Ding JH and He XY contributed to the design of the study and revised the manuscript. Hu CS participated in the design of the study and drafted the manuscript along with Ou XM. All authors read and approved the final manuscript.

\section{Authors' information}

Professor Hu Chaosu, Chairman of the Committee of Nasopharyngeal Carcinoma, Chinese Anti-Cancer Association (CACA). Vice-chairman of Department of Radiation Oncology, Fudan University Shanghai Cancer Center.

\section{Ethics approval and consent to participate}

Institutional Review Board approval was obtained for this retrospective review of patients.

\section{Consent for publication}

Not applicable.

\section{Competing interests}

The authors declare that they have no competing interests.

\section{Publisher's Note}

Springer Nature remains neutral with regard to jurisdictional claims in published maps and institutional affiliations.

\section{Author details}

'Department of Radiation Oncology, Fudan University Shanghai Cancer Center, Shanghai, China. ${ }^{2}$ Department of Oncology, Shanghai Medical College, Shanghai, China. ${ }^{3}$ Department of Diagnostic Radiation, Fudan University Shanghai Cancer Center, Shanghai, China.

Received: 8 March 2017 Accepted: 10 August 2017

\section{Published online: 18 August 2017}

\section{References}

1. Lee AW, Ng WT, Chan LL, Hung WM, Chan CC, Sze HC, Chan OS, Chang AT, Yeung RM. Evolution of treatment for nasopharyngeal cancer-success and setback in the intensity-modulated radiotherapy era. Radiother Oncol. 2014; 110(3):377-84

2. Sun X, Su S, Chen C, Han F, Zhao C, Xiao W, Deng X, Huang S, Lin C, Lu T. Long-term outcomes of intensity-modulated radiotherapy for 868 patients with nasopharyngeal carcinoma: an analysis of survival and treatment toxicities. Radiother Oncol. 2014;110(3):398-403.

3. Lin S, Pan J, Han L, Guo Q, Hu C, Zong J, Zhang X, Lu JJ. Update report of nasopharyngeal carcinoma treated with reduced-volume intensity-modulated radiation therapy and hypothesis of the optimal margin. Radiother Oncol. 2014;110(3):385-9.

4. Ou X, Zhou X, Shi Q, Xing X, Yang Y, Xu T, Shen C, Wang X, He X, Kong L, et al. Treatment outcomes and late toxicities of 869 patients with nasopharyngeal carcinoma treated with definitive intensity modulated radiation therapy: new insight into the value of total dose of cisplatin and radiation boost. Oncotarget. 2015:6(35):38381-97.

5. Zeng L, Tian YM, Sun XM, Chen CY, Han F, Xiao WW, Deng XW, Lu TX. Late toxicities after intensity-modulated radiotherapy for nasopharyngeal carcinoma: patient and treatment-related risk factors. Br J Cancer. 2014;110(1):49-54.

6. Kam MK, Leung SF, Zee B, Chau RM, Suen JJ, Mo F, Lai M, Ho R, Cheung KY, Yu BK, et al. Prospective randomized study of intensity-modulated radiotherapy on salivary gland function in early-stage nasopharyngeal carcinoma patients. J Clin Oncol. 2007:25(31):4873-9.

7. Hsiung CY, Ting HM, Huang HY, Lee CH, Huang EY, Hsu HC. Parotid-sparing intensity-modulated radiotherapy (IMRT) for nasopharyngeal carcinoma: preserved parotid function after IMRT on quantitative salivary scintigraphy, and comparison with historical data after conventional radiotherapy. Int J Radiat Oncol Biol Phys. 2006;66(2):454-61.

8. Pow EH, Kwong DL, McMillan AS, Wong MC, Sham JS, Leung LH, Leung WK. Xerostomia and quality of life after intensity-modulated radiotherapy vs. conventional radiotherapy for early-stage nasopharyngeal carcinoma: initial report on a randomized controlled clinical trial. Int J Radiat Oncol Biol Phys. 2006:66(4):981-91.

9. Gensheimer MF, Liao JJ, Garden AS, Laramore GE, Parvathaneni U. Submandibular gland-sparing radiation therapy for locally advanced oropharyngeal squamous cell carcinoma: patterns of failure and xerostomia outcomes. Radiat Oncol. 2014;9:255.

10. Eisbruch A. Reducing xerostomia by IMRT: what may, and may not, be achieved. J Clin Oncol. 2007;25(31):4863-4.

11. Zhang F, Cheng YK, Li WF, Guo R, Chen L, Sun Y, Mao YP, Zhou GQ, Liu X, Liu LZ, et al. Investigation of the feasibility of elective irradiation to neck level Ib using intensity-modulated radiotherapy for patients with nasopharyngeal carcinoma: a retrospective analysis. BMC Cancer. 2015;15:709.

12. Murdoch-Kinch CA, Kim HM, Vineberg KA, Ship JA, Eisbruch A. Dose-effect relationships for the submandibular salivary glands and implications for their sparing by intensity modulated radiotherapy. Int J Radiat Oncol Biol Phys. 2008;72(2):373-82.

13. Saarilahti K, Kouri M, Collan J, Kangasmaki A, Atula T, Joensuu H, Tenhunen M. Sparing of the submandibular glands by intensity modulated radiotherapy in the treatment of head and neck cancer. Radiother Oncol. 2006;78(3):270-5.

14. Kam MK, Teo PM, Chau RM, Cheung KY, Choi PH, Kwan WH, Leung SF, Zee B, Chan AT. Treatment of nasopharyngeal carcinoma with intensitymodulated radiotherapy: the Hong Kong experience. Int J Radiat Oncol Biol Phys. 2004;60(5):1440-50

15. Lin S, Pan J, Han L, Zhang X, Liao X, Lu JJ. Nasopharyngeal carcinoma treated with reduced-volume intensity-modulated radiation therapy: report on the 3-year outcome of a prospective series. Int J Radiat Oncol Biol Phys. 2009;75(4):1071-8

16. Gregoire V, Ang K, Budach W, Grau C, Hamoir M, Langendijk JA, Lee A, Le QT, Maingon P, Nutting $C$, et al. Delineation of the neck node levels for head and neck tumors: a 2013 update. DAHANCA, EORTC, HKNPCSG, NCIC CTG, NCRI, RTOG, TROG consensus quidelines. Radiother Oncol. 110(1):172-81.

17. van den Brekel MW, Stel HV, Castelijns JA, Nauta JJ, van der Waal I, Valk J, Meyer CJ, Snow GB. Cervical lymph node metastasis: assessment of radiologic criteria. Radiology. 1990;177(2):379-84.

18. King AD, Ahuja AT, Leung SF, Lam WW, Teo P, Chan YL, Metreweli C. Neck node metastases from nasopharyngeal carcinoma: MR imaging of patterns of disease. Head Neck. 2000;22(3):275-81.

19. Lam WW, Chan YL, Leung SF, Metreweli C. Retropharyngeal lymphadenopathy in nasopharyngeal carcinoma. Head Neck. 1997;19(3):176-81.

20. Ng SH, Chang JT, Chan SC, Ko SF, Wang HM, Liao CT, Chang YC, Yen TC Nodal metastases of nasopharyngeal carcinoma: patterns of disease on MRI and FDG PET. Eur J Nucl Med Mol Imaging. 2004;31(8):1073-80.

21. Ho FC, Tham IW, Earnest A, Lee KM, Lu JJ. Patterns of regional lymph node metastasis of nasopharyngeal carcinoma: a meta-analysis of clinical evidence. BMC Cancer. 2012;12:98.

22. Wang $X$, Hu C, Ying $H$, He X, Zhu G, Kong L, Ding J. Patterns of lymph node metastasis from nasopharyngeal carcinoma based on the 2013 updated consensus guidelines for neck node levels. Radiother Oncol. 2015:115(1):41-5.

23. Yuan G, Zheng X, Zhu X, Wang Z, Song W, Zhang H, Sha Z. Risk factors of level Ib lymphadenopathy in nasopharyngeal carcinoma. Nan Fang Yi Ke Da Xue Xue Bao. 2014;34(7):983-7.

24. Yi W, Li X, Liu Z, Jiang C, Niu D, Xia Y. A risk score model for the metastasis of level Ib lymph node based on the clinicopathological features of nasopharyngeal carcinoma in a large sample. Mol Clin Oncol. 2014;2(5):789-97.

25. Pan WR, Suami H, Corlett RJ, Ashton MW. Lymphatic drainage of the nasal fossae and nasopharynx: preliminary anatomical and radiological study with clinical implications. Head Neck. 2009;31(1):52-7.

26. Chen J, Ou D, He X, Hu C. Sparing level Ib lymph nodes by intensity-modulated radiotherapy in the treatment of nasopharyngeal carcinoma. Int J Clin. 2014;19(6):998-1004.

27. Lan M, Huang Y, Chen CY, Han F, Wu SX, Tian L, Zheng L, Lu TX. Prognostic value of cervical nodal necrosis in nasopharyngeal carcinoma: analysis of 1800 patients with positive cervical nodal metastasis at MR imaging. Radiology. 2015;276(2):536-44.

28. Li WF, Sun Y, Mao YP, Chen L, Chen YY, Chen M, Liu LZ, Lin AH, Li L, Ma J. Proposed lymph node staging system using the international consensus guidelines for lymph node levels is predictive for nasopharyngeal 
carcinoma patients from endemic areas treated with intensity modulated radiation therapy. Int J Radiat Oncol Biol Phys. 2013;86(2):249-56.

29. Guo Q, Pan J, Zong J, Zheng W, Zhang C, Tang L, Chen B, Cui X, Xiao Y, Chen $Y$, et al. Suggestions for lymph node classification of UICC/AJCC staging system: a retrospective study based on 1197 nasopharyngeal carcinoma patients treated with intensity-modulated radiation therapy. Medicine. 2015;94(20):e808.

Submit your next manuscript to BioMed Central and we will help you at every step:

- We accept pre-submission inquiries

- Our selector tool helps you to find the most relevant journal

- We provide round the clock customer support

- Convenient online submission

- Thorough peer review

- Inclusion in PubMed and all major indexing services

- Maximum visibility for your research

Submit your manuscript at www.biomedcentral.com/submit
Biomed Central 